\title{
Evidências de validade da Bateria Informatizada de Linguagem Oral com prova de raciocínio'
}

\author{
Evidence of validity of Computerized Oral Language \\ Battery with reasoning assessment
}

\author{
Maria Cristina Rodrigues Azevedo JOLY2 \\ Nayane Martoni PIOVEZAN²
}

\begin{abstract}
Resumo
Este estudo buscou relacionar a compreensão da linguagem oral com a habilidade de raciocinar, visando evidenciar validade correlacional para a Bateria Informatizada de Linguagem Oral por meio da associação com a Escala de Maturidade Mental Colúmbia. Participaram do estudo 82 alunos com idade entre 5 e 9 anos ( $M=7,05$; DP=1,25), sendo 47,6\% do sexo feminino. Frequentavam o pré $(31,7 \%)$ e a 1a $(32,9 \%)$ e 2a $(35,4 \%)$ séries do Ensino Fundamental de escola pública do interior paulista. Aplicou-se coletivamente a Bateria e individualmente a Escala de Maturidade Mental Colúmbia. A Análise de Variância, por série, indicou diferença significativa para as provas de Compreensão Morfossintática, Sequência Lógica, Organização Lógico-Verbal e escore total da Bateria. A análise de regressão revelou que o raciocínio geral representa 29\% das habilidades necessárias para um bom desempenho na Bateria. Verificou-se correlação baixa entre escores totais da Bateria e do Colúmbia, porém muito significativa. Constataram-se evidências de validade correlacional e de critério, para a Bateria.
\end{abstract}

Unitermos: Avaliação psicológica informatizada. Compreensão oral. Ensino fundamental.

\begin{abstract}
The aim of this study was to relate the understanding of oral language to the ability to reason, in order to seek correlational validity evidence of the Computerized Oral Language Battery (Bateria Informatizada de Linguagem Oral) by association with the Columbia Mental Maturity Scale. The participants were 82 schoolchildren between 5 and 9 years of age $(M=7.05, S D=1.25)$. They attended pre-school (31.7\%), 1st (32.9\%) and $2^{\text {nd }}(35.4 \%)$ grades of elementary public school in São Paulo and $47.6 \%$ of whom were girls. The Bateria Informatizada de Linguagem Oral was applied collectively in the school informatics laboratory and the Columbia Mental Maturity Scale, individually. Analysis of Variance for the series, indicated significant statistics differences for the scores of Morphosyntactic prove, Logical sequence prove, Logical Organization prove and for the total score of Bateria Informatizada de Linguagem Oral. Regression analysis revealed that general reason represents 29\% of the skills needed to perform well in Bateria Informatizada de Linguagem Oral. There was low, but very significant correlation between total scores of Bateria Informatizada de Linguagem Oral and Columbia Mental Maturity Scale. These results indicated validity evidences to Bateria Informatizada de Linguagem Oral.
\end{abstract}

Uniterms: Computer-based test. Comprehension. Elementary school.

$\boldsymbol{\nabla} \mathbf{v} \boldsymbol{\nabla}$

I Apoio: Conselho Nacional de Desenvolvimento Científico e Tecnológico.

2 Universidade São Francisco, Pós-Graduação Stricto Sensu em Psicologia. R. Alexandre Rodrigues Barbosa, 45, 13251-900, Itatiba, SP, Brasil. Correspondência para/Correspondence to: M.C.R.A. JOLY. E-mail:<cristina@trtec.com.br>. 
A linguagem vem sendo foco de interesse de diversos pesquisadores desde a década de 1960 (Clay, 1998). Atualmente, os estudos abordam diferentes perspectivas teóricas, nas várias áreas de conhecimento (e.g. psicologia, educação, linguística), o que lhes confere uma abordagem interdisciplinar (Boone \& Plante, 1994; Ellis, 1995; Borges \& Salomão, 2003; Tellez, 2004).

Enquanto habilidade cognitiva, segundo Sternberg (2000), a linguagem tem seis propriedades. A primeira é a propriedade comunicativa, que permite a comunicação entre pessoas que compartilham do mesmo código linguístico. A segunda é a natureza arbitrariamente simbólica, no que se refere à relação entre um símbolo e seu referente, quer seja uma ideia, um objeto, um processo, uma relação ou uma descrição. A terceira é que a linguagem é regularmente estruturada, ou seja, um significado é dado a partir de organizações específicas de símbolos. A quarta propriedade é a organização da linguagem em níveis múltiplos (sons, palavras, frases). A quinta característica refere-se à capacidade de gerar símbolos inéditos, considerando os limites da própria estrutura linguística. A sexta e última está relacionada ao dinamismo da linguagem, em suas múltiplas formas.

Nesse sentido, a habilidade para se comunicar efetivamente envolve, dentre outras coisas, compreensão do que se ouve ou se lê, bem como adequação de conteúdo do que se expressa. Isso porque a linguagem tem um polo receptivo e outro expressivo. O polo receptivo refere-se aos canais de entrada, que não comportam apenas a audição e a compreensão da linguagem falada, mas também a visão e a compreensão da linguagem escrita. O polo expressivo está relacionado aos canais de saída, que são os relativos à fonação ou articulação verbal e à escrita (Flanagan, Ortiz, Alfonso \& Mascolo, 2002; Sternberg, 2000; Gil, 2002).

Dificuldades para compreender o código da linguagem falada (o que as palavras significam) indicam problema no polo receptivo. Se há pouca habilidade para aplicar as regras da linguagem (como combinar as letras e formar novas palavras, ou como organizar uma frase ou discurso em função das variáveis sintáticas, semânticas e pragmáticas) para comunicar ideias e pensamentos, o polo expressivo pode estar comprometido. A deficiência receptiva e a expressiva podem

500 ocorrer de forma independente ou relacionada
(American Speech-Language-Hearing Association, 2007). Sumariando, o polo receptivo possibilita a compreensão, enquanto o polo expressivo viabiliza a comunicação.

Em assim sendo, a compreensão da linguagem oral é um processo cognitivo relativo ao polo receptivo da linguagem, que envolve a compreensão do significado das palavras, frases e textos, a interpretação da informação e sua relação com os conhecimentos prévios, a construção de uma representação mental e sua aplicação. O desenvolvimento da linguagem oral não ocorre somente quando a criança ingressa no ensino formal, mas, segundo Brandão e Spinillo (1998), é uma habilidade que se desenvolve principalmente entre quatro e oito anos de idade e tende a se tornar automática, com ampliação de vocabulário e estabelecimento das relações lógicas entre as informações.

A aquisição e desenvolvimento da linguagem oral ocorrem informalmente a partir daquilo que a criança ouve e fala em sua interação diária com seus pais, pares e outros adultos com quem convive. A leitura de livros infantis, o acesso/uso de materiais de leitura e escrita e a frequência à escola de Educação Infantil, dentre outras, são atividades indicadas para ampliar o vocabulário e a compreensão da linguagem oral (McTavish, 2007).

A revisão da literatura sobre o tema indica que a linguagem oral tem forte correlação com o vocabulário, a consciência fonológica e a nomeação do alfabeto, o que a situa como uma das habilidades preditoras de desempenho tanto para a aquisição quanto para a fluência da leitura e da escrita (Ambruster, Fran Lehr \& Osborn, 2003; Snow, 2002). Desse modo, a detecção de problemas relativos ao campo da linguagem oral permite o desenvolvimento de intervenções logo em seu início, prevenindo posteriores problemas no campo da leitura e escrita (França, Wolff, Moojen \& Rotta, 2004; Hage, Joaquim, Carvalho, Padovani \& Guerreiro, 2004; Salles \& Parente, 2006).

Ao lado dessas premissas teóricas, cabe destacar que, de acordo com Flavell, P.H. Miller e A.S. Miller (1999), a linguagem oral é usada como uma propriedade representante do conhecimento, e, assim, possibilita que se estabeleça uma relação próxima entre a capacidade linguística e a capacidade intelectual geral. Isso porque está relacionada à inteligência cristalizada, que inclui o 
conhecimento de fatos, ideias e conceitos, bem como o uso do raciocínio já aprendido em novas situações (Primi, 2005)

A avaliação da compreensão da linguagem oral adicionada à mensuração de habilidades relacionadas (por exemplo: maturidade mental, inteligência, entre outros) permite uma verificação mais ampla da capacidade, assim possibilitando intervenções que minimizem os problemas encontrados, consideradas as variáveis de status (idade, série e tipo de escola). Isto posto, visando atingir tal intento, foi desenvolvida a Bateria Informatizada de Linguagem Oral (BILO) (Joly, 2006a). Assim, o objetivo do presente estudo é avaliar as características psicométricas da BILO, por meio da associação com a Escala de Maturidade Mental Colúmbia (EMMC).

O primeiro estudo da BILO foi realizado por Joly, Martins, Souza, Istome e Santos (2008), com 119 alunos entre 5 e 10 anos, matriculados na última etapa da Educação Infantil e na $1^{\text {a }}$ e $2^{\text {a }}$ séries do Ensino Fundamental. Antes disso, dois professores e dez alunos de cada série responderam ao instrumento e o avaliaram no tocante à linguagem, vocabulário e imagens. Não se identificou dificuldade na compreensão das instruções, assim como no reconhecimento das imagens e no vocabulário utilizado. Verificaram-se, apenas, dificuldades para a execução das instruções relativas ao uso do computador. Por esse motivo, criou-se um tutorial para orientar os respondentes quanto à utilização do computador e também para assegurar que, a partir daí, seu desempenho não sofresse essa influência.

Visando buscar evidências de validade de construto, Joly et al. (2008) realizaram uma análise fatorial com informação completa por prova, com rotação PROMAX. Constatou-se que as provas de Interpretação de Histórias (IH), Completar Parágrafos (CP) e Completar Histórias $(\mathrm{CH})$ possuíam itens que pouco contribuíam para o total da respectiva prova, os quais foram então retirados. Buscaram-se também os índices de fidedignidade de cada prova, sendo $\mathrm{KR} 20=0,78$ para Compreensão Morfossintática (CM), KR20=0,90 para Sequência Lógica (SQ), KR20=0,64 para Organização Lógico-Verbal (OLV), KR20=0,85 para Interpretação de Histórias (IH), KR20=0,79 para Completar Frases (CF), KR20=0,78 para Completar Parágrafos (CP) e KR20=0,97 para Completar Histórias (CH).
A correlação entre linguagem oral e vocabulário foi o objetivo do estudo de Almeida e Joly (2008), em que 106 alunos com idade entre 5 e 8 anos responderam à BILO (Joly, 2006) e ao Teste de Vocabulário por Imagens Peabody (TVIP) (F.C. Capovilla \& A.G.S. Capovilla, 1997). Os participantes estavam matriculados na última etapa da Educação Infantil e na $1^{\text {a }}$ e $2^{a}$ séries do Ensino Fundamental. O desempenho no TVIP associou-se significativamente às provas de Compreensão Morfossintática $(r=0,35 ; p=0,000)$, Sequência Lógica $(r=0,39 ; p<0,000)$, Organização Lógico-Verbal $(r=0,40 ; p=0,000)$, Interpretação de Histórias $(r=0,30 ; p=0,002)$, Completar Frases $(r=0,24 ; p=0,012)$, Completar Parágrafos $(r=0,30 ; p=0,001)$, Compreensão de Histórias $(r=0,32 ; p=0,002)$ e com o escore total da BILO $(r=0,43 ; p=0,000)$. Desse modo, o estudo evidenciou validade convergente para a BILO, assim como permitiu verificar que, apesar de o vocabulário compor a base estrutural necessária para o entendimento na realização da BILO, esta difere do TVIP enquanto recurso avaliativo.

Ainda neste estudo, as autoras procuraram verificar se a BILO discriminava o desempenho dos respondentes em função do nível de vocabulário apontado pelo TVIP. Para isso, os participantes foram divididos em dois grupos, sendo o primeiro composto por alunos com escore superior a 78,5 pontos, e o segundo com escore inferior a 57,5 pontos. O teste $t$ de Student apontou diferença significativa entre os grupos de participantes $(t[40]=-3,36 ; p=0,02)$. A média de pontuação na BILO para os participantes do grupo com nível superior de vocabulário $(M=121,80 ; D P=14,54)$ foi maior que a média dos participantes com nível inferior $(M=105,50$; $D P=16,87)$. Assim, foi demonstrada a correlação entre os construtos e confirmadas evidências de validade de critério por grupo extremo para a BILO. A análise de variância realizada por meio da Análise de Variância (Anova) identificou que a BILO apresenta validade quanto aos critérios de idade e série escolar, possibilitando verificar a progressão do desempenho dos alunos de acordo com as duas variáveis.

Outro estudo realizado também com a Bateria Informatizada de Linguagem Oral foi conduzido por Issa (2008), a fim de buscar evidência de validade para o instrumento por meio da correlação. Os dados sobre atenção foram obtidos a partir das informações dadas pelos professores na Escala de Transtornos de Déficit de 
Atenção e Hiperatividade para Professores (Benzick, 2000). Os alunos frequentavam o pré, a 1 a série e a $2^{a}$ de escolas públicas do interior paulista. Encontraram-se correlações significativas com as provas da BILO que envolviam de organização lógica ( $r=-0,20, p=0,021$ para $\mathrm{SL}$ e $r=-0,23, p=0,009$ para OLV), indicando que os respondentes acabam não se atentando a detalhes que permitiriam a ordenação das cenas numa sequência e a posterior recordação das informações para a realização da tarefa, por exemplo. Os resultados revelaram que a BILO discrimina o desempenho do respondente em função da série e idade, sendo esta uma evidência de validade de critério para a Bateria. Como conclusão, o estudo evidenciou validade divergente e de critério para a BILO.

Ferracini (2005) buscou validar instrumentos de avaliação da linguagem oral para pré-escolares controlando o efeito da inteligência não-verbal por meio do escore padronizado da EMMC. As análises de variância e covariância utilizando o estanino da EMMC como covariante revelaram efeito significativo da idade sobre os escores totais em todos os instrumentos (Teste de vocabulário por Imagens Peabody, Teste de nomeação, Teste de Repetição de Palavras e Pseudopalavras, Teste de Discriminação Fonológica, Prova de Consciência Fonológica por Produção Oral), com exceção de dois deles (Prova de Consciência Sintática e Lista de Avaliação do Vocabulário Expressivo). Desse modo, verifica-se que outros estudos buscaram evidências de validade para instrumentos que mensuram a linguagem oral, por meio da relação com a habilidade de raciocinar globalmente.

Em revisão de literatura, encontram-se diversos estudos que utilizaram a EMMC para avaliar o raciocínio. Porém, no que se refere a sua relação com a linguagem, percebe-se que, em muitos deles, o escore na Escala serviu como critério para a avaliação de outras variáveis interligadas, como consciência fonológica e de linguagem (A.G.S. Capovilla \& F.C. Capovilla, 2000; Kamhi, Lee \& Nelson, 1985; Pestun, 2005), avaliação da linguagem (Moyle, Weismer, Berman \& Wimberley, n.d.), danos relativos à linguagem e suas variações (Kleeck, Woude \& Hammett, 2006; Leonard et al., 2007; McGregor, Newman, Reilly \& Capone, 2002; Rice, Taylor \& Zubrick, 2008; Thordardottir \&Weismer, 2002).

A proficiência na habilidade de raciocinar, adqui502 rida pelo investimento da capacidade geral em expe- riências de aprendizagem, é baseada na linguagem (Burgemeister, Blum \& Lorge, 1971; Flavell et al., 1999). Dada a carência de estudos que associem a habilidade de raciocinar, em seu sentido mais geral, com a linguagem oral, o objetivo do presente estudo foi relacioná-las, visando buscar evidências de validade para a BILO, por meio da correlação com a EMMC.

\section{Método}

\section{Participantes}

Participaram do presente estudo 82 alunos com idade entre 5 e 9 anos ( $M=7,05 ; D P=1,25)$, sendo 47,6\% do sexo feminino e $52,4 \%$ do masculino. Dentre eles, $31,7 \%$ frequentavam o pré, 32,9\% a 1 a série e 35,4\% a $2^{\text {a }}$ série do Ensino Fundamental de uma escola pública do interior paulista, em amostra composta por conveniência.

\section{Instrumentos}

A Bateria Informatizada de Linguagem Oral (BILO) (Joly, 2006a), tem por objetivo avaliar a compreensão da linguagem oral receptiva em alunos da Educação Infantil à segunda série do Ensino Fundamental (Ciclo I) por meio de um instrumento informatizado. Conta com sete provas referentes a avaliação morfossintática, sequência lógica e compreensão de frases, parágrafos e histórias.

As provas relativas à compreensão da linguagem (considerando sua estrutura em frases, parágrafos e histórias) foram elaboradas conforme o Sistema Orientado de Cloze (SOC) (Joly, 2006b). Este orienta a organização de um texto para avaliar a compreensão em leitura a partir de critérios específicos - número de palavras, omissão de vocábulos, tamanho de lacunas e opções de resposta - visando determinar níveis diferenciados de dificuldade de compreensão. O critério definido para as provas da BILO foi a omissão de um substantivo por frase, o qual deveria ser identificado dentre três opções, uma das quais constituída por palavra de outra classe gramatical.

Especificamente para a compreensão de histórias, há uma prova usando o SOC (Completar História) e outra baseada em questões de interpretação (Inter- 
pretação de Histórias). As instruções gerais e específicas de cada prova e item são apresentados oralmente pelo programa e estão também impressas usando letra bastão.

Prova 1. Compreensão Morfossintática (CM):Tem por objetivo avaliar a relação entre um vocábulo e sua representação gráfica, revelando a compreensão do significado. É constituída por 12 itens, sendo os dois primeiros de exemplo. Cada item é formado por duas orações com um termo integrante que pode ser uma logomarca, substantivo, adjetivo, verbo ou advérbio, a ser compreendido pelo respondente. Assim, a tarefa do respondente é escolher, dentre as três opções, a que melhor representa o conteúdo das orações. A pontuação máxima é de 20 pontos.

Prova 2. Sequência Lógica (SL): Visa identificar a organização lógica e temporal do respondente para estímulos visuais organizados em cenas, cujo conjunto sequenciado resulta em uma história. É constituída por 12 itens, sendo os dois primeiros de exemplo. Cada item é formado por uma série de cenas que, organizadas em uma sequência com começo, meio e fim, adquirem significado de uma história. A tarefa do respondente é organizar as cenas de cada série, de modo que haja relação de causa e efeito e de contiguidade entre elas. Orienta-se exclusivamente pelos estímulos visuais, sendo esta a única prova da BILO que não agrega estímulos orais às imagens para sua execução. A pontuação máxima é de 20 pontos.

Prova 3. Organização Lógico-Verbal (OLV): Avalia a compreensão receptiva do conteúdo de uma história apresentada por completo em vídeo e oralmente, por meio de uma organização lógica de cenas que representam esse conteúdo. É constituída por 12 itens, sendo os dois primeiros de exemplo. Cada item é composto por uma história e por uma série de cenas. Após ouvir a história, o respondente deve organizar as cenas de cada série, de acordo com a sequência lógica do conteúdo visual e verbal. O número de itens por série varia. A pontuação máxima é de 20 pontos.

Prova 4. Interpretação de História (IH): Tem por objetivo avaliar a compreensão receptiva da história, por meio de questões de múltipla escolha. É composta por um exemplo e três descrições apropriadas à faixa etária dos respondentes. A tarefa do respondente é assistir ao vídeo da história e escolher uma opção por item. Todos os itens referem-se à compreensão literal da história. A pontuação máxima é de 20 pontos.

Prova 5. Completar Frases (CF): É avaliada a compreensão de palavras organizadas em frases, sendo um dos substantivos omitido. É constituída por doze itens, sendo dois de exemplo. A tarefa do respondente é identificar a alternativa que melhor dá sentido à frase. A pontuação máxima é de 20 pontos.

Prova 6. Completar Parágrafos (CP): Tem por objetivo avaliar a compreensão de palavras organizadas em três frases que compõem um parágrafo, sendo o substantivo o critério de omissão. É constituída por cinco parágrafos com temas diferentes, sendo um de exemplo. A tarefa do respondente é identificar a alternativa que melhor dá sentido à frase. A pontuação máxima é de 20 pontos.

Prova 7. Completar Histórias (CH): Avalia a compreensão oral de histórias, sendo a primeira constituída por 50 vocábulos e a segunda por 100 vocábulos. Foi eliminado um substantivo a cada dez vocábulos. A primeira história possui quatro omissões, e a segunda, seis omissões. Cada história completa é apresentada em forma de vídeo (imagens com áudio) e, em seguida, na tela acompanhada do respectivo áudio e com as opções de resposta por item. A tarefa do respondente é completar a história com as opções apresentadas depois de assistir ao vídeo. A pontuação máxima é de 20 pontos.

Optou-se por respostas apresentadas como desenhos simples feitos apenas com linhas, sem detalhes. Estes foram selecionados considerando-se símbolos, significados e contextos familiares à criança. As instruções gerais e específicas de cada prova e item são apresentadas por meio do áudio da Bateria e também visualizadas na tela do monitor do computador. Cada tela contém apenas um item e, após a escolha da resposta, o participante é solicitado a confirmá-la antes de passar para o próximo item. Cada prova possui seu conjunto de itens e instruções. Os critérios de correção são por item, por prova e gerais.

Estudos sobre as características psicométricas da BILO foram conduzidos por Almeida e Joly (2008), Joly et al. (2008) e Issa (2008), revelando evidências de validade de conteúdo, construto e critério.

A Escala de Maturidade Mental Colúmbia (Alves \& Duarte, 1994) fornece uma estimativa da capacidade 
de raciocínio geral de crianças com idade de 3 anos e 6 meses a 9 anos e 11 meses. A EMMC possui 92 itens e 3 exemplos, sendo composta por 8 escalas ou níveis de idade que determinam os itens a serem aplicados conforme a faixa etária. Tais itens consistem de uma série de três a cinco desenhos, impressos em cartão, geralmente dentro do campo de experiência da maioria das crianças investigadas, mesmo daquelas com experiências ambientais limitadas. A tarefa solicitada é que a criança selecione, dentre as figuras, a que é diferente ou que não se relaciona com as demais, apontando sua escolha. Para fazê-lo, ela deve estabelecer uma regra de organização das figuras, de modo a excluir apenas uma. As bases para discriminação variam desde a percepção de diferenças grosseiras na cor, tamanho ou forma, ao reconhecimento de relações muito sutis em pares de figuras, de modo a excluir uma delas. Os itens estão organizados em ordem de dificuldade aproximada. A aplicação dura de 15 a 20 minutos, incluindo instrução prévia à criança, usando-se 3 itens como exemplo. 0 resultado é obtido por meio do número de itens respondidos corretamente, comparado ao desempenho de crianças da mesma faixa etária, que foram estudadas para a normatização do teste.

Um estudo de validade (Alves \& Duarte, 2001) desse instrumento foi realizado correlacionando-o com o teste de Matrizes Progressivas de Raven. Obtiveram-se correlações entre os dois testes, encontrando-se 0,67 para crianças de 6 anos, 0,56 para crianças de 8 anos e 0,60 para o grupo total. Quanto à precisão, estudos encontraram coeficientes relativos aos itens variando entre 0,82 a 0,93, com um coeficiente mediano de 0,87.

\section{Procedimentos}

A investigação descrita no presente artigo foi autorizada pelo Comitê de Ética em Pesquisa da Universidade São Francisco, de acordo com Protocolo CAEE: 0172.0.142.000.08. Foi solicitada também autorização formal da escola, bem como dos pais ou responsáveis pelas crianças participantes, os quais assinaram previamente o Termo de Consentimento Livre e Esclarecido.

Foi aplicada, primeiramente, a BILO, de forma coletiva no laboratório de informática, com duração de 30 minutos, em média, em grupos de até 10 respon-

504 dentes, sob orientação de um aplicador e um auxiliar.
Posteriormente, em data previamente agendada com a instituição de ensino, foi realizada a aplicação da Escala de Maturidade Mental Colúmbia, de forma individual, com duração aproximada de 20 minutos.

\section{Resultados}

A partir dos objetivos do presente estudo, foi realizada a estatística descritiva dos subtestes da BILO e do escore total da EMMC, considerando as variáveis de gênero, série e idade. Avaliaram-se as provas por meio da correlação de Pearson entre os escores da BILO e da EMMC. Foram executadas também as análises relativas aos escores de cada prova da BILO.

Considerando as médias dos escores totais da BILO e do EMMC em relação ao sexo dos participantes desta amostra, pode-se perceber, de um lado, que o desempenho dos participantes foi superior ao ponto médio, tanto para cada prova ou escore total da BILO quanto para o EMMC. De outro lado, as meninas tiveram média de acertos maior nos dois instrumentos (Tabela 1). Em função disso, foi realizado um teste $t$ de Student para verificar possíveis diferenças quanto aos escores dos subtestes da BILO e Colúmbia em relação à variável gênero. Os resultados da análise indicaram não haver diferença estatisticamente significativa para os subtestes Compreensão Morfossintática ( $t[80]=-0,250 ; p=0,804)$, Sequência Lógica ( $t[80]=1,146 ; p=0,255)$, Organização Lógico-Verbal $(t[80]=0,912 ; p=0,365)$, Interpretação de Histórias $(t[80]=1,166 ; p=0,247)$, Completar Frases $(t[80]=1,026 ; p=0,308)$, Completar Parágrafos $(t[80]=0,750$; $p=0,455)$, Completar Histórias $(t[80]=-0,124 ; p=0,902)$ e para o escore total da BILO (t[80]=0,780; $p=0,438)$, assim como para o escore total do EMMC (t[80]=0,634; $p=0,528)$.

A Analise de Variância (ANOVA), por série, indicou diferença estatisticamente significativa para os subtestes Compreensão Morfossintática $(F[2,79]=4,87 ; p=0,01)$, Sequência Lógica $(F[2,79]=6,27 ; p=0,00)$, Organização Lógico-Verbal $(F[2,79]=3,16 ; p=0,04)$ e também para o escore total $(F[2,79]=5,03 ; p=0,00)$ da BILO. Observou-se que os alunos das séries iniciais obtiveram pontuação inferior aos alunos das séries posteriores, em todos os escores.

Quanto à idade, a ANOVA indicou diferença estatisticamente significativa para as provas CM (F[4, 
$77]=2,92 ; p=0,02), S L(F[4,77]=3,20 ; p=0,01)$ e CF $(F[4,77]=3,27 ; p=0,01)$. A análise post hoc de Tukey diferenciou os alunos de 5 anos daqueles com 8 e 9 anos somente para a prova $\mathrm{CM}$, sendo que os mais velhos tiveram médias maiores que os mais novos. Para a SL e CF não houve distinção. Quanto aos escores totais dos instrumentos, diferenças marginalmente significativas foram verificadas para a BILO ( $F[4,77]=2,40 ; p=0,57)$ e para o EMMC (F[4,77] $=2,38 ; p=0,58)$.

Realizou-se a correlação de Pearson entre escore total da BILO com escore total do EMMC. Pode-se observar associação baixa, porém muito significativa entre os construtos medidos pelos instrumentos (Tabela 2).
Nota-se que não houve associações significativas entre os escores do Pré e da 1a série, com exceção do subteste IH para esta última. Por outro lado, observa-se que os escores da 2a série nos subtestes SL, OLV, CFECH e total da BILO associaram-se de modo significativo ao escore obtido no EMMC, o que pode denotar que, à medida que os alunos ascendem em nível escolar, adquirem maior maturidade mental, característica natural do desenvolvimento.

No total, verifica-se que as associações significativas deram-se com provas da BILO que envolvem, justamente, as habilidades relacionadas ao raciocínio lógico, como exigido na realização do EMMC, quais

Tabela 1. Médias dos escores dos subtestes e escore total da BILO e do EMMC em função do sexo dos participantes. Amparo (SP), 2007.

\begin{tabular}{|c|c|c|c|}
\hline Provas & Sexo & Média & Desvio-Padrão \\
\hline \multirow[t]{2}{*}{ Compreensão morfossintática } & Feminino & 16,28 & 3,11 \\
\hline & Masculino & 16,44 & 2,68 \\
\hline \multirow[t]{2}{*}{ Sequência lógica } & Feminino & 12,64 & 5,32 \\
\hline & Masculino & 11,26 & 5,54 \\
\hline \multirow[t]{2}{*}{ Organização lógico-verbal } & Feminino & 12,81 & 5,12 \\
\hline & Masculino & 11,81 & 4,78 \\
\hline \multirow[t]{2}{*}{ Interpretação de histórias } & Feminino & 10,54 & 1,94 \\
\hline & Masculino & 9,86 & 3,12 \\
\hline \multirow[t]{2}{*}{ Completar frases } & Feminino & 8,10 & 1,52 \\
\hline & Masculino & 7,74 & 1,63 \\
\hline \multirow[t]{2}{*}{ Completar parágrafos } & Feminino & 14,51 & 1,21 \\
\hline & Masculino & 14,19 & 2,46 \\
\hline \multirow[t]{2}{*}{ Completar histórias } & Feminino & 18,79 & 8,54 \\
\hline & Masculino & 19,02 & 8,19 \\
\hline \multirow[t]{2}{*}{ Escore total BILO } & Feminino & 93,68 & 19,02 \\
\hline & Masculino & 90,33 & 19,78 \\
\hline \multirow[t]{2}{*}{ Escore total columbia } & Feminino & 36,03 & 5,81 \\
\hline & Masculino & 35,05 & 7,90 \\
\hline
\end{tabular}

BILO: Bateria Informatizada de Linguagem Oral; EMMC: Escala de Maturidade Mental Columbia.

Tabela 2. Correlação entre os subtestes e escore total da BILO e escore total da EMMC, por série e no total. Amparo (SP), 2007.

\begin{tabular}{lllll}
\hline \multirow{2}{*}{ BILO } & \multicolumn{3}{c}{ EMMC } \\
\cline { 2 - 4 } & Pré & 1a série & 2a série & Total \\
\hline Compreensão morfossintática & $-0,002$ & $-0,230$ & 0,359 & 0,017 \\
Sequência lógica & $-0,060$ & 0,116 & $0,662^{* *}$ & $0,282^{* *}$ \\
Organização lógico-verbal & $-0,002$ & 0,282 & $0,513^{* *}$ & $0,299^{* *}$ \\
Interpretação de histórias & 0,008 & $0,592^{* *}$ & 0,274 & $0,300^{* *}$ \\
Completar frases & 0,273 & $-0,260$ & $0,561^{* *}$ & 0,143 \\
Completar parágrafos & $-0,296$ & $-0,006$ & 0,048 & $-0,101$ \\
Completar histórias & $-0,318$ & 0,187 & $0,553^{* *}$ & $0,215^{*}$ \\
Escore total & $-0,187$ & 0,199 & $0,736^{* *}$ & $0,292^{* *}$ \\
\hline
\end{tabular}

${ }^{*} p<0,05 ;{ }^{* *} p<0,01$. BILO: Bateria Informatizada de Linguagem Oral; EMMC: Escala de Maturidade Mental Columbia. 
sejam a utilização da habilidade cognitiva geral, o conhecimento prévio adquirido (Ambuster et al., 2003; Snow, 2002) e o estabelecimento de uma regra de organização para escolher a resposta (Burgemeister et al., 1971; Flavell et al., 1999; Primi, 2005). A prova SL implica organizar adequadamente as cenas apresentadas para que formem uma história, sendo necessário que o respondente utilize o raciocínio lógico juntamente com o prévio conhecimento que tem sobre o vocabulário presente nas cenas. Já em OLV, o respondente necessita compreender as informações dadas e, a partir disso, organizar as cenas na sequência adequada, fazendo uso da lógica baseada na informação compreendida. Esses achados ratificam os resultados das investigações de Almeida e Joly (2008), A.G.S. Capovilla e F.C. Capovilla (2000), Kamhi et al. (1985) e Pestun (2005).

O raciocínio geral é necessário para que a realização de $\mathrm{H}_{\mathrm{H}} \mathrm{CH}$ seja proficiente, já que, no primeiro caso, a tarefa consiste em demonstrar a compreensão da história apresentada por meio de respostas a questões de múltipla escolha, e no segundo, é preciso que o respondente tenha a compreensão oral da história para que faça a escolha da melhor alternativa que a completará de forma lógica. Em ambas as provas, a criança deve decodificar sons em símbolos e utilizar a habilidade cognitiva geral para a escolha da resposta, partindo da compreensão ocorrida.

Levando em conta que a linguagem oral é usada como uma propriedade representante do conhecimento, que estabelece uma relação próxima entre a capacidade linguística e a capacidade intelectual geral (Flavell et al., 1999), foi realizada a análise de regressão múltipla. Esta revelou o raciocínio geral como representante de 29\% das habilidades necessárias para um bom desempenho na BILO.

\section{Discussão}

A análise do desempenho dos participantes, quer na BILO quer no EMMC, indicou desempenho superior à média, revelando que não se constataram dificuldades, nem da perspectiva da avaliação específica da compreensão da linguagem oral (Sternberg, 2000), nem desta como medida indireta e relacionada à capacidade cognitiva (Flavell et al. 1999; Primi, 2005). Tal constatação permite hipotetizar que os participantes prova- velmente não apresentarão dificuldades para aquisição da linguagem escrita, de acordo com a perspectiva teórica de Snow (2002) e de Ambuster et al. (2003).

Constataram-se diferenças estatisticamente significativas para desempenho na BILO em função do desenvolvimento e da escolaridade, o que revela evidências de validade de critério para a BILO (Urbina, 2007). Tais resultados corroboram os obtidos nos estudos de Almeida e Joly (2008), Joly et al. (2008) e Issa (2008) com a BILO.

Cabe destacar que, de acordo com Brandão e Spinillo (1998), as crianças da faixa etária investigada no presente estudo apresentam uma ampliação notória de sua capacidade linguística. Há que se considerar também, segundo McTavish (2007), que a frequência à escola de educação infantil pode ter auxiliado para tal competência linguística.

A associação baixa e muito significativa entre os escores de cada prova da BILO com o desempenho geral no EMMC em função da série e com amostra total também atribui evidências de validade para a BILO (Urbina, 2007).

Considerando que o resultado revela um desenvolvimento apropriado da linguagem oral e também da capacidade de raciocínio geral, observa-se que a BILO possibilita discriminar o desempenho dos estudantes pelo nível de escolaridade, fortalecendo a evidência de validade de critério já verificada pela análise da ANOVA por série. No tocante às associações constatadas para o desempenho da amostra total, verificou-se a utilização da habilidade cognitiva geral associada ao conhecimento prévio adquirido (Ambuster et al., 2003; Snow, 2002) e o estabelecimento de uma regra de organização para escolher a resposta (Burgemeister et al., 1971; Flavell et al., 1999; Primi, 2005). Focaliza-se o fato de que o raciocínio geral é necessário para que a realização de $\mathrm{H}$ e $\mathrm{CH}$ seja proficiente, já que, em ambas as provas, a criança deve decodificar sons em símbolos e utilizar a habilidade cognitiva geral para a escolha da resposta, partindo da compreensão da história.

Acrescente-se a isso que a análise de regressão ratificou a importância de avaliar tanto a linguagem oral quanto as habilidades gerais de raciocínio a fim de evitar problemas quando da aquisição formal da linguagem escrita, como propõem França et al. (2004), Hage et 
al., (2004) e Salles e Parente (2006). Assim, foi possível confirmar, neste estudo, a estreita relação entre os fenômenos psicológicos pesquisados e atingir os objetivos propostos.

\section{Considerações Finais}

Os resultados observados possibilitaram verificar evidência de validade convergente para a BILO por meio da correlação com a EMMC, pois o raciocínio geral representa parte das habilidades necessárias para a realização da tarefa proposta pela BILO. Verificou-se também validade de critério, pois a BILO possibilitou discriminar o desempenho dos estudantes em função de sua escolaridade e desenvolvimento.

Constatou-se, também, que a avaliação da compreensão da linguagem oral para a amostra em questão pode ser um indicador da capacidade cognitiva adquirida a partir do processo de aprendizagem, dada a natureza da BILO como prova de desempenho.

Por fim, faz-se necessário apontar as limitações do presente estudo quanto à amostra investigada e, portanto, considerá-lo com as restrições e limites intrínsecos. Sugerem-se novas investigações com amostras mais diversificadas, considerando-se a avaliação de um histórico de vida dos participantes que melhor caracterize seu desenvolvimento linguístico, permitindo a identificação de outras variáveis relevantes.

\section{Referências}

Almeida, A. R., \& Joly, M. C. R. A. (2008). Estudo correlacional entre a Bateria Informatizada de Linguagem Oral (BILO) e Peabody. In L. Almeida, C. Machado, M. Gonçalves \& A. P. P. Noronha. Avaliação psicológica: formas e contextos (pp.1-13). Braga: Psiquilibrios.

Alves, I. C. B., \& Duarte J. L. M. (1994). Escala de Maturidade Mental de Colúmbia: padronização brasileira. São Paulo: Casa do Psicólogo.

Alves, I. C. B., \& Duarte, J. L. M. (2001). Escala de maturidade mental Colúmbia: manual para aplicação e interpretação. São Paulo: Casa do Psicólogo.

Ambruster, B. B., Fran Lehr, M. A., \& Osborn, J. (2003). A child becomes a reader: proven ideas for parents from researchkindergarten through grade three. Jessup: EdPubs.

American Speech-Language-Hearing Association. (2007). What is language? What is speech? Retrieved on August 14, 2009, from <http://www.asha.org/public/speech/ development/language_speech.htm>.
Benzick, E. B. P. (2000). Manual da escala de transtorno de déficit de atenção/hiperatividade. São Paulo: Casa do Psicólogo.

Boone, D. R., \& Plante, E. (1994). Comunicação humana e seus distúrbios ( $2^{\mathrm{a}} \mathrm{ed}$ ). Porto Alegre: Artes Médicas.

Borges, L. C., \& Salomão, N. M. R. (2003). Aquisição de linguagem: considerações da perspectiva da interação social. Psicologia: Reflexão e Crítica, 16 (2), 327-336.

Burgemeister, B., Blum, L., \& Lorge, I. (1971). Columbia Mental Maturity Scale. New York: Harcout, Brace \& Ovanovich.

Brandão, A. C. P., \& Spinillo, A. G. (1998). Aspectos gerais e específicos na compreensão de textos. Psicologia: Reflexão e Crítica, 11 (2), 253-272.

Capovilla, A. G. S.; \& Capovilla, F. C. (2000). Efeitos do treino de consciência fonológica em crianças com baixo nível sócio-econômico. Psicologia: Reflexão e Crítica, 13 (1), 7-24.

Capovilla, F. C., \& Capovilla A. G. S. (1997). Desenvolvimento lingüístico da criança dos dois aos seis anos: Ciência Cognitiva: Teoria, Pesquisa e Aplicação, 1 (1), 53-380.

Clay, M. M. (1998). By different paths to common outcomes. Portland: Stenhouse Publishers.

Ellis, W. E. (1995). Leitura, escrita e dislexia: uma análise cognitiva ( $2^{\mathrm{a}}$ ed.) Porto Alegre: Artmed.

Ferracini, F. (2005). Evidências de validade de instrumentos para avaliação de linguagem oral em pré-escolares. Dissertação de mestrado não-publicada, Programa de PósGraduação Stricto Sensu em Psicologia, Universidade São Francisco, Itatiba.

Flanagan, D. P., Ortiz, S. O., Alfonso, V. C., \& Mascolo, J. T. (2002). The achievement test - desk reference. Boston: Allyn and Bacon.

Flavell, J. H., Miller, P. H., \& Miller, A. S. (1999). Desenvolvimento cognitivo. Porto Alegre: Artes Médicas.

França, M. P., Wolff, C. L., Moojen, S., \& Rotta, N. T. (2004). Aquisição da linguagem oral: relação e risco para a linguagem escrita. Arquivo de Neuropsiquiatria, 62 (2-B), 469-472.

Gil, R. (2002). Neuropsicologia. São Paulo: Livraria Santos Editora.

Hage, S. R. V., Joaquim, R. S. S., Carvalho, K. G., Padovani, C. R., \& Guerreiro, M. M. (2004). Diagnóstico de crianças com alterações específicas de linguagem oral por meio de escala de desenvolvimento. Arquivos de Neuropsiquiatria, $62(3-A), 649-653$.

Issa, G. M. P. (2008). Estudos de evidências de validade da Bateria Informatizada de Linguagem Oral-BILO. Dissertação de mestrado não-publicada, Programa de Pós-Graduação Stricto Sensu em Psicologia, Universidade São Francisco, Itatiba.

Joly, M. C. R. A. (2006a). Bateria informatizada de linguagem oral: projeto em desenvolvimento. In L. Almeida, C. Machado, M. Gonçalves \& A. P. P. Noronha. Avaliação psicológica: formas e contextos [CD-ROM]. Braga: Psiquilíbrios. 
Joly, M. C. R. A. (2006b). Cloze Oriented System (COS) in an electronic comprehension program and reading attitude in Brazil. In A. Mendéz-Villas, B. G. Pereira, J. M. González \& J. A. M. González (Eds.), Current developments in technology-assisted education (pp.1669-1674). Badajoz: Indugrafic.

Joly, M. C. R. A., Martins, R. X., Souza, A. C. Z., Istome, A. C., \& Santos, C. R. O. A. (2008). Bateria Informatizada de Linguagem Oral (BILO): validade de construto. In L. Almeida, C. Machado, M. Gonçalves \& A. P. P. Noronha (Orgs.), Avaliação psicológica: formas e contextos (pp.121-140). Braga: Psiquilíbrios.

Kamhi, A. G., Lee, R. F., \& Nelson, L. K. (1985). Word, syllable, and sound awareness in language-disordered children. Journal of Speech and Hearing Disorders, 50, 207-212.

Kleeck, A., Woude, J. V., \& Hammett, L. (2006). Fostering literal and inferential language skills in head start preschoolers with language impairment using scripted cook-sharing discussions. American Journal of Speech-Language Pathology, 15, 85-95.

Leonard, L. B., Deevy, P., Kurtz, R., Chorev, L. K., Owen, A., Polite, E., et al. (2007). Lexical aspect and the use of verb morphology by children with specific language impairment. Journal of Speech, Language and Hearing Research, 50 (3), 759-777.

McGregor, K. K., Newman, R. M., Reilly, R. M., \& Capone, N. C. (2002). Semantic representation and naming in children with specific language impairment. Journal of Speech, Language, and Hearing Reasearch, 45 (5), 998-1014.

McTavish, M. (2007) Constructing the big picture: a working class family supports their duaghter's pathways to literacy. The Reading Teacher, 60 (5), 476-485.

Moyle, M. J., Weismer, S. E., Berman, S. S., \& Wimberley, K. (n.d.). Nonword repetition in preschoolers: a comparison of AAE and MAE speakers. Retrieved on May 30, 2009, from <http://convention.asha.org/2007/handouts/1137_
1542Jones_Moyle_Maura_106701_Nov13_2007_Time_ 022331PM.doc>.

Pestun, M. S. V. (2005). Consciência fonológica no início da escolarização e o desempenho ulterior em leitura e escrita: estudo correlacional. Estudos de Psicologia (Natal), 10 (3), 407-412.

Primi, R. (2005). Avaliação componencial informatizada da inteligência fluida. Projeto de Pesquisa não-publicado, Programa de Pós-Graduação Strictu Sensu em Psicologia, Universidade São Francisco, Itatiba.

Rice, M. L., Taylor, C. L., \& Zubrick, S. R. (2008). Language outcomes of 7-year-old children with or without a history of late language emergence at 24 months. Journal of Speech, Language and Hearing Research, 51 (2), 394-407.

Salles, J. F., \& Parente, M. A. M. P. (2006). Funções neuropsicológicas em crianças com dificuldades de leitura e escrita. Psicologia: Teoria e Pesquisa, 22 (2), 153-162.

Snow, C. (2002). Reading for understanding. Santa Monica: RAND.

Sternberg, R. J. (2000). Psicologia cognitiva. Porto Alegre: Artmed.

Tellez, J. A. (2004). La comprensión de los textos escritos y la psicología cognitiva. Madrid: Dykinson.

Thordardottir, E. T., \& Weismer, S. E. (2002). Verb argument structure weakness in specific language impairment in relation to age and utterance length. Clinical Linguistics \& Phonetics, 16 (4), 233-250.

Urbina, S. (2007). Fundamentos da testagem psicológica. Porto Alegre: Artmed.

Recebido em: 11/1/2011

Versão final em: 3/11/2011

Aprovado em: 13/2/2012 